\title{
Il sistema solare tra l'ordine e il caos
}

\author{
di Stefano Marmi \\ (docente di Analisi presso la Scuola Normale Superiore di Pisa)
}

L'errore fecondo di Henri Poincarè segnò il tramonto del sogno che aveva guidato le ricerche dei matematici per oltre due secoli: era impossibile descrivere e prevedere esattamente la dinamica di ogni sistema deterministico, qualunque fosse il suo stato iniziale. Il problema dei tre corpi non era integrabile, cioè la sua soluzione non poteva essere ricondotta a un procedimento analitico-algebrico di "quadrature". I| procedimento utilizzało classicamente per risolvere le equazioni differenziali più semplici avrebbe consentito, non solo in linea di principio ma anche computazionalmente, la previsione con una precisione prestabilita della posizione futura dei pianeti.

Il problema della stabilità delle orbite planetarie nel sistema solare, che si sperava chiuso da tempo, si riproponeva quindi integralmente. Successivamente ai lavori di Poincaré, G.D. Birkhoff dimostrò come le instabilità nei moti planetari fossero davvero possibili e che la struttura delle orbite nello spazio delle fasi del problema degli n corpi era formidabilmente complessa. Un importante progresso fu compiuto nel 1954 da A.N. Kolmogorov con l'enunciato del teorema di persistenza delle orbite quasi-periodiche nei sistemi hamiltoniani quasi-integrabili. Dimostrato successivamente da V.I. Arnol'd e J. Moser, divenne celebre come Teorema KAM. II risultato di Kolmogorov costituiva una rivincita del paradigma della stabilità e della prevedibilità delle orbite nei problemi di Meccanica celeste: i moti quasi-periodici sono infatti indistinguibili dai moti periodici se la precisione delle osservazioni è fissata e finita. Tuttavia, a differenza di un genuino moto periodico, nel caso quasi-periodico il periodo osservato cresce illimitatamente al migliorare detla precisione. Al contrario dei comportamenti genuinamente instabili e caotici previsti nei lavori di Poincarè e di Birkhoff, le orbite quasi-periodiche non manifestano però nessuna dipendenza sensibile dai dati iniziali e la costruzione di effemeridi affidabili sarebbe rimasta possibile, almeno in qualche caso. Kolmogorov, che conosceva bene sia i lavori di Poincaré che quelli di Birkhoff, suggerì che la struttura tipica delle orbite nello spazio delle fasi di un sistema hamiltoniano prevedesse la coesistenza di un comportamento instabile per un insieme topologicamente generico di condizioni iniziali, e un comportamento stabile e quasi-periodico per un insieme probabilisticamente generico di condizioni iniziali.

L'instabilità per un insieme di condizioni iniziali topologicamente generico implica che, anche se il moto di un pianeta o di un asteroide è regolare, una perturbazione arbitrariamente piccola della sua posizione o velocità è sufficiente a metterlo su un'orbita irregolare e caotica (diffusione di Arnol'd). II processo con cui questo tipo di diffusione si può manifestare è tuttavia piuttosto lento (teorema di Nekhoroshev) e questo insieme di risultati, benchè di natura teorica e non direttamente applicabile a modelli realistici del sistema solare, rinforzò la convinzione che la scala di tempi necessaria affinché si manifesti un comportamento caotico nella dinamica del sistema solare dovesse essere molto lunga in confronto con la sua età. Le simulazioni numeriche della dinamica del sistema solare si scontrano con la necessità di integrare le equazioni di Newton per il problema degli $n$ corpi per miliardi di anni con un passo di integrazione temporale molto breve, al più di qualche ora. II pianeta più veloce, Mercurio, compie infatti il suo moto orbitale in meno di tre mesi e impiegare passi di integrazione più lunghi condurrebbe ad errori negli schemi numerici tali da inficiarne le conclusioni.

Nel 1990 J. Laskar aggirò il problema sostituendo al sistema di equazioni di Newton il cosiddetto sistema secolare introdotto da Lagrange. II vantaggio principale di questo procedimento consiste nell'eliminazione delle variabili angolari veloci (corrispondenti al moto imperturbato dei pianeti lungo le ellissi kepleriane). Si rinuncia cosi al calcolo delle effemeridi dei pianeti, limitando lo studio alle variabili che descrivono la dinamica della lenta deformazione delle orbite dei pianeti nel tempo per effetto delle perturbazioni reciproche. Le variabili utilizzate da Laskar furono in realtà introdotte da Poincaré: il moto imperturbato dei pianeti corrisponde semplicemente ad un punto di equilibrio per la dinamica del sistema secolare. Poiché in queste variabili le soluzioni kepleriane corrispondono all'assenza di moto, è possibile utilizzare un passo di integrazione molto più lungo di quello necessario per la simulazione numerica diretta del sistema: 500 anni anziché poche ore. In questo modo fu possibile per la prima volta integrare numericamente un modello realistico del sistema solare su una scala di tempo dell'ordine di $10^{10}$ anni. I risultati furono sorprendenti: le orbite dei pianeti del sistema solare interno Mercurio, Venere, Terra e Marte) sono caotiche con una scala temporale per I'instabilità, misurata dal tempo di Ljapunov, dell'ordine di 5 milioni di anni. Una conseguenza pratica di un valore cosi basso (su una scala di tempi astronomica) del tempo di Ljapunov è l'impossibilità del calcolo di un'effemeride planetaria per i pianeti interni: in circa 100 milioni di anni un errore nella posizione iniziale di 15 metri può crescere fino a dare un'indeterminazione della posizione su una scala di 150 milioni di chilometri, cioè della stessa grandezza della distanza attuale della Terra dal Sole. La situazione è ben diversa nel caso del sistema solare esterno: le orbite dei pianeti maggiori (Giove, Saturno, Urano e Nettuno) sembrano invece essere ben descritte da movimenti quasi-periodici. L'attività di ricerca per dimostrare rigorosamente questo risultato è molto intensa e tra i protagonisti ci sono gli italiani Antonio Giorgilli e Ugo Locatelli.

\section{Bibliografia}

Marmi S., "Chaotic behaviour in the Solar System (following J. Laskar)", in Astérisque, 2000, 266, pp. 113-136; Chenciner A., "Poincare and the Three-Body Problem", in Séminaire Poincaré (Bourbaphy)XVI, 2012, pp. 45-133 (http:// wuw. bourbaphy.fr/novembre2012.html). 